\title{
Short-time dynamics of percolation observables
}

\author{
Wanderson G. Wanzeller \\ Instituto de Física Teórica, Universidade Estadual Paulista, Rua Pamplona 145, 01405-900 São Paulo, SP, Brazil \\ Tereza Mendes \\ Instituto de Física de São Carlos, Universidade de São Paulo, C.P. 369, 13560-970 São Carlos, SP, Brazil \\ Gastão Krein \\ Instituto de Física Teórica, Universidade Estadual Paulista, Rua Pamplona 145, 01405-900 São Paulo, SP, Brazil
}

(Received 19 May 2006; published 28 November 2006)

\begin{abstract}
We consider the critical short-time evolution of magnetic and droplet-percolation order parameters for the Ising model in two and three dimensions, through Monte Carlo simulations with the (local) heat-bath method. We find qualitatively different dynamic behaviors for the two types of order parameters. More precisely, we find that the percolation order parameter does not have a power-law behavior as encountered for the magnetization, but develops a scale (related to the relaxation time to equilibrium) in the Monte Carlo time. We argue that this difference is due to the difficulty in forming large clusters at the early stages of the evolution. Our results show that, although the descriptions in terms of magnetic and percolation order parameters may be equivalent in the equilibrium regime, greater care must be taken to interpret percolation observables at short times. In particular, this concerns the attempts to describe the dynamics of the deconfinement phase transition in QCD using cluster observables.
\end{abstract}

DOI: 10.1103/PhysRevE.74.051123

PACS number(s): 64.60.Ak, 05.10.Ln, 12.38.Aw, 64.60.Ht

\section{INTRODUCTION}

It is often useful to map a physical phase transition into the geometric problem of percolation, e.g., in order to gain a better understanding of how the transition is induced in the system (see, e.g., Ref. [1]). This mapping is obtained through a suitable definition of cluster in terms of the system's variables and parameters. In the case of the Ising model, such physical (droplet) clusters were introduced by Coniglio and Klein (based on the prescription by Kasteleyn and Fortuin) [2] and the mapping is well understood [3]. This also holds for the so-called $n$-vector models [4]. The correct cluster definition is of special interest in the description of more complex phase transitions, such as the quark-deconfinement transition in finite-temperature QCD, for which the physical order parameter is not yet established. In this case there is still no satisfactory definition for a "droplet" cluster, even in the simpler pure-gauge case [5].

When equilibrium properties are investigated, the descriptions of the spin-model phase transition in terms of the magnetic order parameter or the percolation order parameter are indeed equivalent and one finds the same critical exponents (see, for example, Ref. [6] and references therein). The same may or may not be true for the dynamical evolution of the different types of order parameters, although in principle one might expect to find equivalence for dynamic quantities as well, in particular for the behavior at short times. We recall that the short-time behavior of the magnetic order parameter $M$ is described by a scale-free expression, in terms of a power law (see, for instance Refs. [7-9]).

The study of the dynamic critical behavior of a percolation order parameter with respect to the (Glauber) Monte Carlo evolution might be of relevance for understanding non-equilibrium effects in hot $\mathrm{QCD}$, such as the effects due to the heating and cooling of matter produced in heavy-ion collisions. Indeed, the possible connection between the deconfinement transition in QCD and the percolation phenomenon [10] has received renewed attention in recent years [5] and the dynamics of cluster observables has been investigated using hysteresis methods [11]. Note that the QCD phase transition is predicted to fall into the Ising universality class in the pure two-color $[\mathrm{SU}(2)]$ case and that the chiral phase transition in the two-flavor full-QCD case is expected to be in the universality class of the (continuous-spin) fourvector model [12-14]. This motivates the connection between the percolation transitions for spin models and the (dynamic) behavior at the QCD phase transition. We thus consider here the short-time dynamics of the two- and threedimensional Ising model and focus on the dynamic critical behavior of the percolation order parameter. (Preliminary results of our study were presented in $[15,16]$.) Note that the dynamic behavior of cluster observables has been considered for the droplet clusters in Ising and Potts models in various studies (see, e.g., $[17,18]$ ), but mostly for the so-called cluster numbers $n_{s}$-which denote the average number of clusters (per lattice site) containing $s$ sites each-and not for the percolation order parameter.

Here we investigate the dynamic critical behavior of the (zero-field) Ising model

$$
\mathcal{H}=-J \sum_{\langle i, j\rangle} S_{i} S_{j},
$$

where $J$ is positive and each $S_{i}= \pm 1$, for short Monte Carlo times $t$, using the (local) heat-bath algorithm. We measure the magnetization $M=(1 / V) \sum_{i} S_{i}$ and we consider two different definitions for the percolation order parameter. Indeed, given a definition for a cluster on the lattice, one may consider as the order parameter in percolation theory the strength of the percolating cluster, defined for each configu- 
ration by the relative volume of the infinite cluster. (In particular, in the droplet picture for the Ising model, this would give zero for temperatures above the critical one.) On a finite lattice, one might consider the volume of the infinite cluster to be the one of the spanning cluster, or zero when there is no percolating cluster (i.e., above the critical temperature). Note that a spanning cluster is a set of spins connected from the first to the last row of the lattice in at least one of its space directions. Alternatively, one can also consider the relative volume of the largest cluster (see, e.g., chapter 3 in [3]). We denote these two definitions for the percolation order parameter respectively by $\Omega$ (in the case of the spanning cluster) and $\Omega^{\prime}$ (in the case of the largest cluster, which is not necessarily a percolating one).

In this work we compare the behaviors of the two types of quantities just described (i.e., percolation or magnetic order parameters) as functions of $t$. As shown below, we find that whereas the magnetic order parameter displays a power-law increase with $t$, the data for the percolation order parameters $\Omega$ and $\Omega^{\prime}$ are well fitted, respectively, by a diffusion and by a behavior similar to the one of nucleation processes in firstorder transitions. In both cases the time scales-respectively called $\tau$ and $\tau^{\prime}$-are related to the relaxation time to equilibrium. Indeed, we show that $\tau$ and $\tau^{\prime}$ diverge as $L^{z}$ when the lattice side $L$ tends to infinity, where $z \approx 2$ is the dynamic critical exponent of the heat-bath algorithm [19].

\section{SHORT-TIME (MONTE CARLO) DYNAMICS}

Using renormalization-group theory, it can be shown $[9,20]$ that the early time evolution of an order parameter (e.g., the magnetization $M$ ) already displays universal critical behavior, given by

$$
M\left(t, \epsilon, m_{0}\right)=b^{-\beta / \nu} \mathcal{M}\left(t b^{-z}, \epsilon b^{1 / \nu}, m_{0} b^{x_{0}}\right),
$$

where $m_{0}$ is the initial magnetization, $\epsilon \equiv\left(T-T_{c}\right) / T_{c}, \mathcal{M}$ is a universal function, and $b$ is a scale factor, which can be taken equal to $t^{1 / z}$. We thus expect for $T=T_{c}$ and small $m_{0}$ a powerlaw behavior at early times

$$
M(t)_{\epsilon \rightarrow 0} \sim m_{0} t^{\theta},
$$

with $\theta=\left(x_{0}-\beta / \nu\right) / z$. In principle, we would assume that the two percolation order parameters $\Omega$ or $\Omega^{\prime}$ defined above should have a similar behavior.

The heat-bath dynamics consist in choosing the two possible directions of each Ising spin according to the exact conditional probability given by its nearest neighbors. Each spin $S_{i}$ is chosen "up" or "down," respectively, with probability $p_{i}$ or $1-p_{i}$, where

$$
p_{i}=\frac{1}{1+\exp \left(-2 \beta J \sum_{j} S_{j}\right)},
$$

$\beta=1 / k_{B} T$, and the sum is over nearest neighbors of $S_{i}$. After a certain number of iterations the spin configuration obeys the Boltzmann distribution. In the heat-bath method, since the updates are local, this transient time becomes considerably large at criticality.

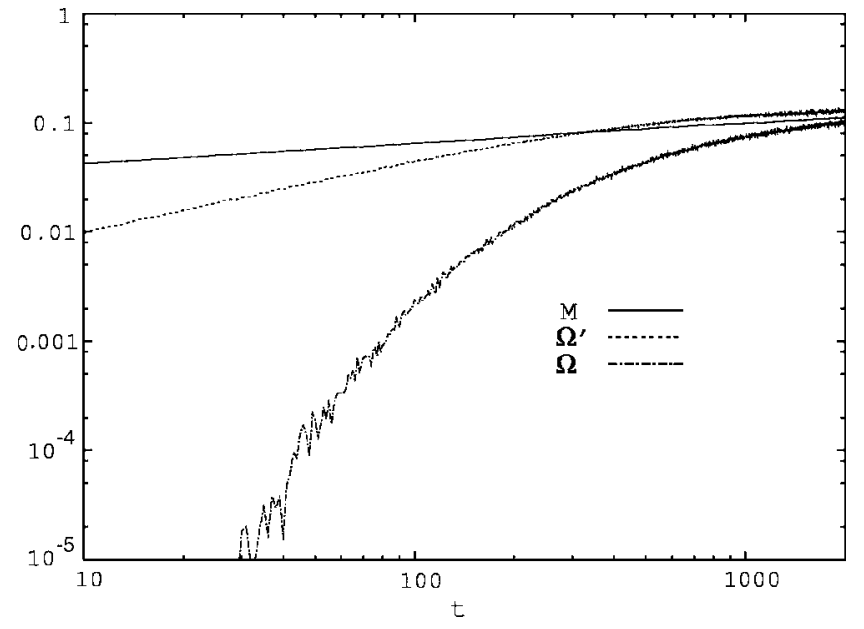

FIG. 1. Plot of the early time evolution of the magnetic $(M)$ and percolation $\left(\Omega, \Omega^{\prime}\right)$ order parameters for the two-dimensional case. Data are shown for $m_{0}=0.02$ and $L=200$. Note the logarithmic scale on both axes.

The Fortuin-Kasteleyn clusters are obtained from the Ising-model Hamiltonian by writing the partition function as

$$
\mathcal{Z}=\sum_{\{S\}} \sum_{\{n\}}\left\{\prod_{\langle i, j\rangle}^{n_{i j}=1} p_{i j} \delta_{S_{i} S_{j}}\right\}\left\{\prod_{\langle i, j\rangle}^{n_{i j}=0}\left(1-p_{i j}\right)\right\},
$$

where $p_{i j}=1-\exp (-2 J \beta)$ is the probability of having a link between two nearest-neighbor sites of equal spin value. This link is represented by $n_{i j}$ and determines the clusters that will be associated with percolation at the critical temperature [21]. Note that the above defined clusters are used in the Swendsen-Wang algorithm [22] to perform global moves in which the spins in a cluster are flipped together. Here we only use these clusters to calculate percolation observables, whereas the dynamics are given are local heat-bath updates, as described above.

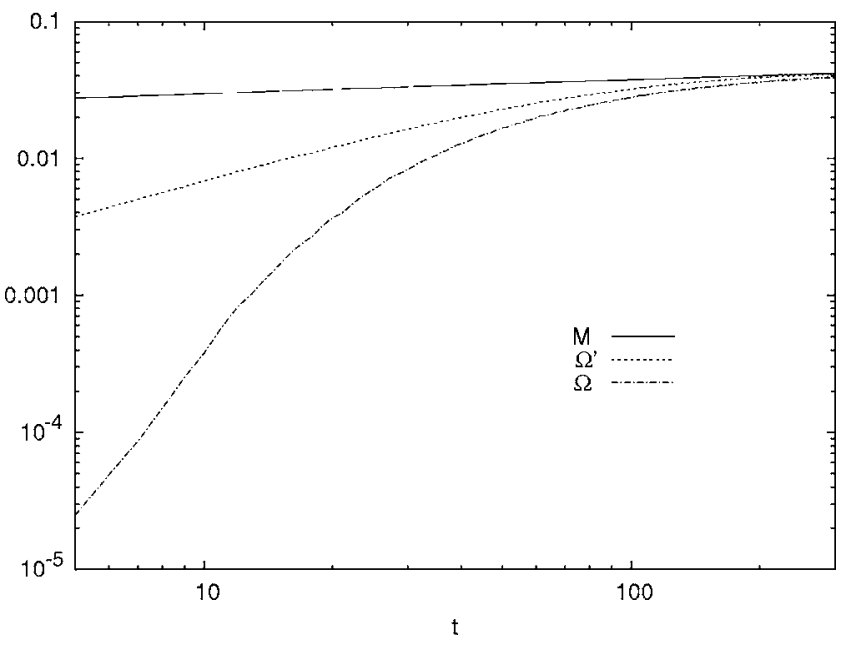

FIG. 2. Plot of the early time evolution of the magnetic $(M)$ and percolation $\left(\Omega, \Omega^{\prime}\right)$ order parameters for the three-dimensional case. Data are shown for $m_{0}=0.02$ and $L=64$. Note the logarithmic scale on both axes. 
TABLE I. Fits of $\Omega(t)$ to the form $A \exp (-\tau / t)$. Data for the case $m_{0}=0.02$. The fit intervals are chosen as shown in the second column, whereas the errors in the fit parameters are adjusted to account for the fluctuations in these parameters for slightly different fit intervals.

\begin{tabular}{lcccc}
\hline \hline Volume & $\Delta t$ & $\mathrm{~A}$ & $\tau$ & $\chi^{2} / \mathrm{DOF}$ \\
\hline $100^{2}$ & $18-83$ & $0.061(1)$ & $85.5(9)$ & 0.97 \\
$125^{2}$ & $28-110$ & $0.062(1)$ & $134(1)$ & 0.98 \\
$150^{2}$ & $30-150$ & $0.063(1)$ & $192(2)$ & 0.91 \\
$200^{2}$ & $41-240$ & $0.061(1)$ & $334(3)$ & 0.69 \\
$24^{3}$ & $3-100$ & $0.0366(3)$ & $5.9(3)$ & 0.57 \\
$32^{3}$ & $10-120$ & $0.0397(5)$ & $11.7(6)$ & 0.42 \\
$48^{3}$ & $15-120$ & $0.0446(4)$ & $28.4(4)$ & 0.58 \\
$64^{3}$ & $20-130$ & $0.0467(3)$ & $51.6(3)$ & 0.68 \\
\hline \hline
\end{tabular}

\section{NUMERICAL RESULTS}

In order to study the short-time dynamics we simulate at $T=T_{c}$ and force the system to have a random configuration with initial magnetization $m_{0}$. This is done by flipping the appropriate number of randomly located spins of a completely disordered (infinite-temperature) configuration. We let the system evolve in time and look for power-law behavior of the order parameters $M, \Omega$, and $\Omega^{\prime}$ as functions of the (Monte Carlo) time. Each temporal sequence is generated from a different random seed, i.e., each sequence has a different initial spin configuration. The time history is then obtained from an average over all the generated sequences.

We have studied the two- and three-dimensional cases, performing Monte Carlo simulations, respectively, with 50000 and 40000 seeds, for several initial magnetizations $m_{0}$ and several lattice volumes, using the heat-bath algorithm. We consider periodic boundary conditions. Note that in order to compare the percolation order parameters $\Omega$ and $\Omega^{\prime}$ to $M$ we consider the volumes of clusters of "up" spins with positive sign and volumes of clusters of "down" spins with negative sign when taking the average over the seeds.

We obtain that a power-law fit works very well for $M$, yielding the literature value [23] for the exponent $\theta$. However, as can be seen in Figs. 1 and 2, the percolation order parameters $\Omega$ and $\Omega^{\prime}$ do not show a power-law behavior, being consistent with exponential behaviors in terms of $t / \tau$, thus having $\tau$ as a time scale. As verified below, the exponential behaviors are different for $\Omega$ and $\Omega^{\prime}$, but $\tau$ is in both cases directly related to the relaxation time to equilibrium. We thus find the surprising result that although the equilibrium behaviors of $M, \Omega$, and $\Omega^{\prime}$ are equivalent, the different types of order parameters show qualitatively different dynamic critical behavior.

More precisely, the behavior of $\Omega$ is given in the two- and three-dimensional cases by

$$
\Omega(t)=A \exp (-\tau / t),
$$

as can be seen from Table I. On the other hand, the behavior of $\Omega^{\prime}$ is given by

$$
\Omega^{\prime}(t)=B\left\{1-\exp \left[-\left(t / \tau^{\prime}\right)^{\eta}\right]\right\}
$$

as shown in Table II.

Fits of the data to the above forms are shown (for the largest lattices considered) in Figs. 3 and 4, respectively, for the two- and three-dimensional cases. Note that the values of $\chi^{2}$ per degree of freedom (DOF) may be significantly smaller than one, since we perform uncorrelated fits. We see that the departure of the percolation order parameters from the power-law behavior of the magnetization remains even long after the so-called microscopic time [9]. In other words, neither $\Omega$ nor $\Omega^{\prime}$ can be fitted to a power law at short times. In fact, the forms proposed for $\Omega$ and $\Omega^{\prime}$ in Eqs. (6) and (7) are

TABLE II. Fits of $\Omega^{\prime}(t)$ to the form $B\left\{1-\exp \left[-\left\{t / \tau^{\prime}\right)^{\eta}\right]\right\}$. Data for the case $m_{0}=0.02$. The fit intervals are chosen as shown in the second column, whereas the errors in the fit parameters are adjusted to account for the fluctuations in these parameters for slightly different fit intervals.

\begin{tabular}{lccccc}
\hline \hline Volume & $\Delta t$ & $\mathrm{~B}$ & $\tau^{\prime}$ & $\eta$ & $\chi^{2} / \mathrm{DOF}$ \\
\hline $100^{2}$ & $7-100$ & $0.104(5)$ & $82(1)$ & $0.71(1)$ & 0.35 \\
$125^{2}$ & $8-100$ & $0.126(5)$ & $168(14)$ & $0.70(1)$ & 0.23 \\
$150^{2}$ & $7-100$ & $0.115(4)$ & $179(13)$ & $0.72(1)$ & 0.17 \\
$200^{2}$ & $10-100$ & $0.20(1)$ & $380(32)$ & $0.73(1)$ & 0.17 \\
$24^{3}$ & $3-100$ & $0.0349(1)$ & $8.0(1)$ & $0.82(1)$ & 0.42 \\
$32^{3}$ & $5-120$ & $0.0372(2)$ & $15.4(3)$ & $0.83(2)$ & 0.50 \\
$48^{3}$ & $10-130$ & $0.0394(3)$ & $34.4(4)$ & $0.90(1)$ & 0.48 \\
$64^{3}$ & $15-150$ & $0.0402(5)$ & $60.5(4)$ & $0.93(1)$ & 0.56 \\
\hline \hline
\end{tabular}




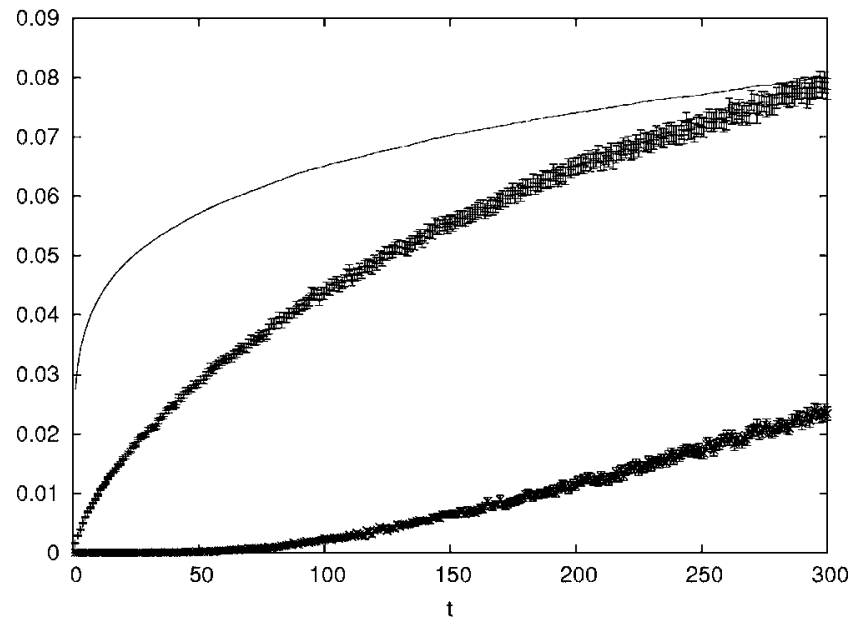

FIG. 3. Data for the two-dimensional case and fits of $\Omega$ (lowest curve) and $\Omega^{\prime}$ (middle curve) according to the forms in Eqs. (6) and (7), respectively. The magnetization (top curve) is also shown, for comparison. Data and fits are shown for $m_{0}=0.02$ and $L=200$. Error bars correspond to one standard deviation.

well fitted by the data in two and three dimensions, for all volumes considered. (We note however that the twodimensional data for $\Omega$ were fitted in [16] considering also a second exponential term, adding a third parameter to this fit.) The above data for $\tau$ and $\tau^{\prime}$ can be fitted to the form $L^{z}$, in the two- and three-dimensional cases separately. In all four cases we find $z \approx 2$. The two time scales can therefore be associated with the relaxation time to equilibrium.

Note that the behavior of $\Omega$ (in two and three dimensions) is a factor $\sqrt{t}$ times the solution of a one-dimensional diffusion equation at a fixed position in space [24]. In fact, the expression for the concentration of diffusion material due to a change in chemical potential along a direction $x$ at a position $x_{0}$ and time $t$ is given by $(C / \sqrt{t}) \exp \left(-x_{0}^{2} / 4 D t\right)$, where $D$ is the diffusion coefficient. Comparing this to the form for $\Omega$ in Eq. (6), we see that if we multiply the percolation order parameter in this case by $\sqrt{t}$ we obtain the diffusive form, identifying $x_{0}^{2} / 4 D$ with $\tau$. Of course, the factor $\sqrt{t}$ corresponds to the diffusion length for a random walk at time $t$. Moreover, the fixed position $x_{0}$ is proportional to $\sqrt{\tau}$, i.e., roughly the length of the lattice (since $\tau \approx L^{z}$ as mentioned above). This connects the occurrence and strength of percolation at time $t$ to the probability that a random walk will reach a length $L$ after $t$ steps.

The behavior of $\Omega^{\prime}$ is similar to the one of the volume fraction of particles in a process of nucleation and growth [24]. We note that such a process occurs in first-order phase transitions, while the physical transition in the two- and three-dimensional Ising models is a continuous one. In particular, the behavior in Eq. (7) is observed in the dynamics of weak first-order phase transitions, as seen, e.g., in Ref. [25]. In that reference, the critical dynamics of a scalar field quenched to a metastable state was investigated with a model- $A$ Langevin equation. In this context, our result for $\Omega^{\prime}$ would illustrate that for the same underlying dynamics (model $A$, in this case of the heath-bath dynamics), the shorttime behavior clearly depends on the specific observable and its initial configuration. It would also be interesting to check

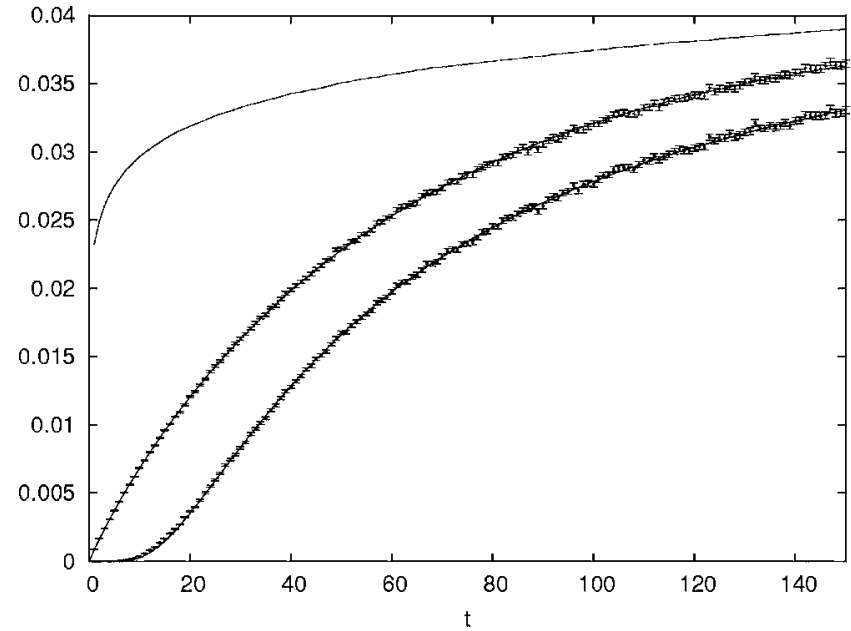

FIG. 4. Data for the three-dimensional case and fits of $\Omega$ (lowest curve) and $\Omega^{\prime}$ (middle curve) according to the forms in Eqs. (6) and (7), respectively. The magnetization (top curve) is also shown, for comparison. Data and fits are shown for $m_{0}=0.02$ and $L=64$. Error bars correspond to one standard deviation.

if the exponent $\eta$ goes to 1 in the limit of very large $L$ in three dimensions (as seems to be suggested by the data in Table II) or to relate the exponents in two and three dimensions to the fractal dimensions associated with the percolating clusters in the two cases.

Regarding the so-called percolation cumulant or percolation probability - taken as 1 if there is percolation and 0 if there is not-we obtain that this quantity does not show a power-law behavior in time, contrary to what is observed for the Binder cumulant [9]. We find that the percolation cumulant is also described by an exponential $\exp (-\tau / t)$, with the prefactor $\sqrt{t}$ in two dimensions and $t^{-0.34}$ in three dimensions.

\section{CONCLUSIONS}

We have investigated numerically the critical heat-bath dynamics for magnetic and percolation order parameters in the Ising model at short Monte Carlo times, starting from a small magnetization $m_{0}$. From our results we see that although the equilibrium behaviors of the magnetization $M$ and of the percolation order parameters $\Omega$ and $\Omega^{\prime}$ are equivalent, the two types of order parameters show qualitatively different dynamic critical behavior at short times. Indeed, whereas the magnetic order parameter $M$ shows a power-law behavior with the exponent $\theta$, one finds that $\Omega$ and $\Omega^{\prime}$ have a time scale, given respectively by $\tau$ in Eq. (6) and $\tau^{\prime}$ in Eq. (7). This time scale seems to be related to the relaxation time of the algorithm used for thermalization.

The short-time behaviors of $\Omega$ and $\Omega^{\prime}$ are well described respectively by diffusion and by a behavior similar to a growth and nucleation process. This may be related to the difficulty in forming a macroscopic cluster at the early stages of the simulation.

\section{ACKNOWLEDGMENTS}

We thank Attilio Cucchieri for helpful discussions. Research was supported by FAPESP, CNPq, and CAPES. 
[1] S. Wenzel, E. Bittner, W. Janke, A. M. J. Schakel, and A. Schiller, Phys. Rev. Lett. 95, 051601 (2005).

[2] P. W. Kasteleyn and C. M. Fortuin, J. Phys. Soc. Jpn. 26, 11 (1969); A. Coniglio and W. Klein, J. Phys. A 13, 2775 (1980).

[3] D. Stauffer and A. Aharony, Introduction to Percolation Theory (Taylor \& Francis, London, 1992).

[4] P. Blanchard, S. Digal, S. Fortunato, D. Gandolfo, T. Mendes, and H. Satz, J. Phys. A 33, 8603 (2000).

[5] H. Satz, Comput. Phys. Commun. 147, 46 (2002); S. Fortunato, J. Phys. A 36, 4269 (2003).

[6] W. G. Wanzeller, A. Cucchieri, G. Krein, and T. Mendes, Braz. J. Phys. 34, 247 (2004).

[7] K. Okano et al., Nucl. Phys. B 485, 727 (1997).

[8] H. J. Luo, L. Schülke, and B. Zheng, Phys. Rev. Lett. 81, 180 (1998).

[9] B. Zheng, Int. J. Mod. Phys. B 12, 1419 (1998).

[10] G. Baym, Physica A 96, 131 (1979).

[11] B. A. Berg et al., Phys. Rev. D 69, 034501 (2004); A. Bazavov, B. A. Berg, and A. Velytsky, ibid. 74, 014501 (2006).

[12] R. D. Pisarski and F. Wilczek, Phys. Rev. D 29, 338 (1984).

[13] J. Engels and T. Mendes, Nucl. Phys. B 572, 289 (2000).
[14] J. Engels et al., Phys. Lett. B 514, 299 (2001).

[15] W. G. Wanzeller, G. Krein, and T. Mendes, in IX Hadron Physics and VII Relativistic Aspects of Nuclear Physics, edited by M. E. Bracco et al., AIP Conf. Proc. 739 (AIP, Melville, 2004), p. 608.

[16] W. G. Wanzeller, T. Mendes, and G. Krein, Braz. J. Phys. 36, 657 (2006).

[17] D. Stauffer, Physica A 186, 197 (1992).

[18] F. Yaşar and M. Aydin, Physica A 255, 430 (1998).

[19] R. da Silva, N. A. Alves, and J. R. D. de Felicio, Phys. Lett. A 298, 325 (2002).

[20] H. K. Janssen, B. Schaub, and B. Schmittmann, Z. Phys. B: Condens. Matter 73, 539 (1989).

[21] R. G. Edwards and A. D. Sokal, Phys. Rev. D 38, 2009 (1988).

[22] R. H. Swendsen and J. S. Wang, Phys. Rev. Lett. 58, 86 (1987).

[23] J.-B. Zang et al., Phys. Lett. A 262, 226 (1999).

[24] R. H. Doremus, Rates of Phase Transformations (Academic Press, New York, 1985).

[25] M. Gleiser, Phys. Rev. Lett. 73, 3495 (1994). 